\title{
ANALYTIC PROPERTIES OF COSINE OPERATORS
}

\author{
S. NELSON AND R. TRIGGIANI ${ }^{1}$
}

\begin{abstract}
Let $A$ be the infinitesimal generator of a strongly continuous cosine operator $C(t)$, hence of the analytic semigroup $S(t)$, on the Banach space $X$. It is proved that the set of analytic vectors for $C(t)$ contains the dense subspace $X_{0}=\bigcup_{0<t} S(t) X$, the containment being in general proper.
\end{abstract}

Let $C(t),-\infty<t<\infty$, be a strongly continuous cosine operator on a Banach space $X$. (For background on cosine operator theory and its relevance to second order abstract differential equations, see [S1], [F1], [K1] $[\mathbf{K} 3]$ and the references therein.) In particular, $C(0)=I$ and for every $t, C(t)=$ $C(-t)$ is a bounded linear operator on $X$. The infinitesimal generator of $C(t)$ is a closed linear operator $A$ with domain $D(A)$ dense in $X$. Moreover, this operator $A$ also generates a strongly continuous semigroup $S(t), t \geqslant 0$ of bounded linear operators on $X$, which may be extended analytically onto the half plane $\operatorname{Re} t>0$. In fact, for $t>0, S(t)$ is given explicitly (see [F2, (5.17)]) by

$$
S(t) x=\frac{1}{\sqrt{\pi t}} \int_{0}^{\infty} e^{-\tau^{2} / 4 t} C(\tau) x d \tau, \quad x \in X
$$

For perspective on the theorem below, we recall that a cosine operator $C(t)$ is uniformly continuous if and only if its infinitesimal generator $A$ is a bounded operator on $X$, in which case $C(t)$ is, in fact, given by the power series $C(t)=\sum_{n=0}^{\infty} A^{n} t^{2 n} /(2 n)$ ! convergent in the uniform operator topology. A proof of the 'only if' part-the nontrivial part-is contained in [K3, pp. 258-260]. An alternate proof is outlined in the remark at the end of the present paper.

A fortiori, analyticity of the map $t \rightarrow C(t)$ on $R$-or, equivalently, [B1, p. 291], [H1, p. 93] of the map $t \rightarrow C(t) x$, for all $x$ in $X$-is possible if and only if the generator $A$ is bounded on $X$. The following result shows that in the genuinely strongly continuous case with $A$ unbounded, there is a dense subspace of $X$ for whose elements $x$ the map $t \rightarrow C(t) x$ is analytic on $R$.

Received by the editors January 11, 1978.

AMS (MOS) subject classifications (1970). Primary 47D05.

Key words and phrases. Cosine operators, semigroups, analytic vectors.

${ }^{1}$ Research partially supported by Air Force O. S. R. under grant AFSOR-77-3338A.

(C) 1979 American Mathematical Society 0002-9939/79/0000-0167/\$02.00 
Namely, the subspace

$$
X_{0} \stackrel{\text { def }}{=} \bigcup_{0<t} S(t) X
$$

which [H1, p. 208] shows to be dense in $X$.

THEOREM. Let $C(t)$ be a strongly continuous cosine operator in the Banach space $X$. Then for every $x \in X_{0}$ and every real number $t$

$$
C(t) x=\sum_{n=0}^{\infty} A^{n} x \frac{t^{2 n}}{(2 n) !} .
$$

Thus, for $x \in X_{0}$, the function $t \rightarrow C(t) x$ can be extended analytically onto the entire complex plane.

Proof. The analyticity of the corresponding semigroup $S(t)$ implies

$$
X_{0} \subset \bigcap_{n=1}^{\infty} D\left(A^{n}\right) \stackrel{\text { def }}{=} D_{\infty}(A)
$$

Hence e.g. [K1]

$$
\frac{d^{2 n} C(t) y}{d t^{2 n}}=A^{n} C(t) y=C(t) A^{n} y, \quad y \in D_{\infty}(A),-\infty<t<\infty,
$$

while the evenness of $C(t)$ implies that the odd derivatives are 0 when $t=0$. Now let $[-T, T]$ be an arbitrary interval. For any $x^{*} \in X^{*}$, the Taylor formula at $t=0$ with Lagrange's remainder is then

$$
x^{*}(C(t) x)-\sum_{n=0}^{N-1} x^{*}\left(A^{n} x\right) \frac{t^{2 n}}{(2 n) !}=x^{*}\left(C(\bar{t}) A^{N} x\right) \frac{t^{2 N}}{(2 N) !}, \quad x \in D_{\infty}(A),
$$

for some $\bar{t}$ in $(0, T)$. By assumption, $x=S(\tau) y$ for some $\tau>0$ and $y \in X$ and the analyticity of $S(\cdot)$ implies [B1, p. 16]

$$
\left\|A^{N} S(\tau)\right\|=\left\|[A S(\tau / N)]^{N}\right\| \leqslant(k N / \tau)^{N}
$$

for some constant $k$, whenever $\tau / N \leqslant 1$, i.e., for $N$ suitably large. By virtue of the standard upper bound on $C(\cdot)$, the Lagrange remainder is majorized in norm by

$$
c\left(\frac{k N}{\tau}\right)^{N} \frac{T^{2 N}}{(2 N) !}<c\left(\frac{k T^{2}}{\tau}\right)^{n} \frac{1}{N !} \quad\left(c=M e^{\omega_{0} T}\left\|x^{*}\right\|\|y\|\right)
$$

which goes to zero as $N \rightarrow \infty$. This establishes weak convergence of the series in question. But weak and strong analyticity of $C(t) x$ are equivalent [B1, p. 291], [H1, p. 93]. That the radius of convergence is $+\infty$ follows also from the Cauchy-Hadamard theorem [H1, p. 96]:

$$
\begin{aligned}
& \varlimsup\left\{\frac{\left\|A^{n} S(\tau) y\right\|}{(2 n) !}\right\}^{1 / 2 n} \leqslant \varlimsup\left\{\left(\frac{k n}{\tau}\right)^{n} \frac{\|y\|}{(2 n) !}\right\}^{1 / 2 n} \\
& =\varlimsup\left\{\frac{1}{2}\left(\frac{k}{\tau n}\right)^{1 / 2}\|y\|^{1 / 2 n}\left[\frac{(2 n)^{2 n}}{(2 n) !}\right]^{1 / 2 n}\right\}=0
\end{aligned}
$$


since, by Stirling's formula, the last factor has limit $e$. Q.E.D.

Now, let $X_{m}$ be the subspace consisting of those $x \in D_{\infty}(A)$ such that (1) holds for all $t \in R$. Obviously $X_{0} \subset X_{m} \subset D_{\infty}(A)$ and we want to settle the questions of proper containment.

To this end, and throughout the rest of the paper, let $X=L_{2}(R), R=$ $(-\infty, \infty), A f=d^{2} f / d \xi^{2}$ (in the sense of distributions) with $D(A)=\{f: f$ and $\left.f^{\prime \prime} \in L_{2}(R)\right\}$. Then $A$, being selfadjoint with spectrum bounded above, generates a strongly continuous cosine operator $C(t)$ as well as a strongly continuous analytic semigroup $S(t)$. $S(t)$ and $C(t)$ as applied to $f(\cdot) \in L_{2}(R)$ are given by

$$
\begin{gathered}
{[S(t) f](\xi)=\frac{1}{\sqrt{4 \pi t}} \int_{-\infty}^{\infty} e^{-(\xi-\sigma)^{2} / 4 t} f(\sigma) d \sigma, \quad t>0,-\infty<\xi<\infty} \\
{[C(t) f](\xi)=[f(\xi+t)+f(\xi-t)] / 2, \quad-\infty<t<\infty}
\end{gathered}
$$

as one sees by considering the solutions to the first and second order equations associated with $A$.

To characterize the functions in $X_{m}$ we introduce $X_{m}(T)$, the subspace of all $x \in D_{\infty}(A)$ such that (1) holds for $-T<t<T$.

Proposition. For $X$ and $A$ as chosen above, an $L_{2}$ function $h$ is in $X_{m}(T)$ if and only if the Fourier transform $\hat{h}$ of $h$ satisfies: $\hat{h}(\omega) \cosh a \omega \in L_{2}(R)$ for all $a \in(0, T)$.

REMARK. The condition on $\hat{h}$ is equivalent to the existence of a complex valued function $H(\xi+i \eta)$, analytic in the strip $\{-\infty<\xi<\infty,-T<\eta<$ $T\}$, equal to $h(\xi)$ when $\eta=0$, and such that $\int_{-\infty}^{\infty}|H(\xi+i \eta)|^{2} d \xi$ is bounded on all intervals $-a \leqslant \eta \leqslant a, a \in(0, T)$. (See [P1, Theorem IV, p. 7] for the difficult half of the equivalence.)

Proof. If the series $\sum_{n=0}^{\infty} A^{n} h t^{2 n} /(2 n)$ ! converges for all $t \in(-T, T)$ then it also converges for all cx. $t$ with $|t|<T$. Fixing $t=i a, a \in(0, T)$, and applying the Fourier transform to the series, we see by Plancherel's theorem that

$$
\sum_{n=0}^{\infty} \omega^{2 n} \hat{h}(\omega) a^{2 n} /(2 n) !=\hat{h}(\omega) \cosh a \omega
$$

represents a function in $L_{2}(R)$.

Conversely, assume $\hat{h}(\omega) \cosh a \omega$ is in $L_{2}(R)$. Let $C_{n}(y)=$ $\sum_{k=0}^{n}(-1)^{k} y^{2 k} /(2 k)$ !. Clearly $\left|C_{n}(y)-\cos y\right| \leqslant \cosh y$ and hence

$$
\left|C_{n}(a \omega) \hat{h}(\omega)-\cos a \omega \hat{h}(\omega)\right|^{2} \leqslant|\hat{h}(\omega) \cosh a \omega|^{2} \text {. }
$$

Since the right-hand side is integrable and the left-hand side approaches 0 as $n \rightarrow \infty$, it follows from Lebesgue's dominated convergence theorem that in the $L_{2}$ norm topology, the series $\sum_{n=0}^{\infty}(-1)^{n} \omega^{2 n} \hat{h}(\omega) a^{2 n} /(2 n)$ ! converges to $\hat{h}(\omega) \cos a \omega$ which by (3) is equal to $\widehat{C(a) h}$. A second application of Plancherel's theorem completes the proof. Q.E.D. 
ExAmples. 1. If $h \neq 0$ is a $C^{\infty}$ function with compact support, then $h \in D_{\infty}(A)$ but $h \notin X_{m}(T)$ for any $T$, since $h$ cannot have an analytic extension $H(\xi+i \eta)$. Thus $X_{m} \varsubsetneqq D_{\infty}(A)$.

2. If $\hat{h}(\omega)=\exp \left\{-|\omega|^{3 / 2}\right\}$ then $h \in X_{m}$ by the proposition. However, since the Fourier transform converts convolution into multiplication, we have, from (2), $[\widehat{S(t) f}](\omega)=e^{-t \omega^{2}} \hat{f}(\omega)$ so that $h \notin X_{0}$. Thus $X_{0} \varsubsetneqq X_{m}$.

REMARK. In his systematic monograph [S1], Sova extensively studies cosine operators. Toward the end of [S1], he refers to [K3] for a proof of the 'only if' part of the result (labeled Fundamental Theorem 3.4): $C(t)$ is uniformly continuous if and only if its generator $A$ is bounded. We wish to point out that an alternative proof of the 'only if' part, can also be given within the framework of results already developed up to that point by Sova. By invoking Lemmas 2.11 and 2.14 of [S1] and applying the same procedure as in the semigroup case [H1, p. 283], there is some $\rho>0$ such that

$$
T(\rho)=\int_{0}^{\rho}(\rho-u) C(u) d u
$$

admits a bounded inverse on $X$. Then

$$
\lim _{h \rightarrow 0} 2 \frac{(C(h)-I) T(\rho)}{h^{2}}=C(\rho)-I
$$

so that $A=(C(\rho)-I) T^{-1}(\rho)$ is bounded. Q.E.D.

REMARK. The present investigation of analytic vectors for cosine operators arose from the study of controllability properties of associated first and second order abstract nonhomogeneous equations in Banach space. The analytic vectors discussed here make the abstract wave equation and the abstract heat equation simultaneously approximately controllable. For details, see [T1].

\section{REFERENCES}

[B1] P. L. Butzer and H. Berens, Semigroups of operators and approximations, Springer-Verlag, Berlin, 1967.

[F1] [F2] H. O. Fattorini, Ordinary differential equations in linear topological spaces. I, II, J. Differential Equations 5 (1968), 72-105; 6 (1969), 50-70.

[H1] E. Hille and R. S. Phillips, Functional analysis and semigroups, Amer. Math. Soc. Colloq. Pulb., vol. 31, Amer. Math. Soc., Providence, R.I., 1958.

[K1] J. Kisinsky, On cosine operator functions and one parameter groups of operators, Studia Math. 44 (1972), 93-105.

[K2] _ On operator-valued solutions of D'Alembert's functional equation. II, Studia Math. 42 (1972), 43-66.

[K3] S. Kurepa, A cosine functional equation in Banach algebras, Acta Sci. Math. (Szeged) 23 (1962), 255-267.

[P1] R. E. A. C. Paley and N. Wiener, Fourier transforms in the complex domain, Amer. Math. Soc. Colloq. Publ., vol. 19, Amer. Math. Soc., Providence, R.I., 1934.

[S1] M. Sova, Cosine operator functions, Rozprawy Mat. 49 (1966), 47 pp.

[T1] R. Triggiani, On the relationship between first and second order controllable systems in Banach space, SIAM J. Control Optimization 16 (1978), 847-859.

Department of Mathematics, Iowa State University, Ames, Iowa 50011 